Pacific Journal of Mathematics

INVARIANT MEASURES AND THE CONVERSE OF HAAR'S 


\title{
INVARIANT MEASURES AND THE CONVERSE OF HAAR'S THEOREM ON SEMITOPOLOGICAL SEMIGROUPS
}

\author{
A. MukherJea AND N. A. Tserpes
}

\begin{abstract}
According to the converse of Haar's theorem, a complete separable metric group which admits a locally finite nonzero $r^{*}$-invariant measure is locally compact. It is proved in this paper that a complete separable metric semitopological (resp. topological) semigroup which admits a finite (resp. possibly infinite) nonzero $r^{*}$ and $l^{*}$-invariant measure is a compact (resp. locally compact) topological group.

The structure of idempotent probability measures and finite $r^{*}$-invariant measures has also been given in the case of locally compact semitopological semigroups. Also it is shown in this paper that for a wide class of nonabelian locally compac $\hat{s}$ semitopological semigroups, admissibility of a twoside invariant measure is equivalent to embeddability in a group.
\end{abstract}

1. Throughout $S$ will be (at least) a Hausdorff semitopological semigroup (an algebraic semigroup with separately continuous multiplication) and $\mu$ a locally finite nonnegative measure (i.e., every point has an open neighborhood of finite measure) defined on the Borel $\sigma$-algebra $\mathscr{B}$ (generated by the open sets) of $S$. We will call $\mu$ nontrivial if $\mu(C)>0$ for at least one compact set $C$. The measure $\mu$ is called inner regular if $\mu(B)$, for any $B \in \mathscr{B}$, can be approximated (from inside) by the measures of compact subsets. It is well known that every nonzero $\mu$ on a complete separable metric or second countable locally compact space is inner regular. We will call $\mu$ $x \in S$.

(a) right invariant if $\mu(C x)=\mu(C)$ for all compact $C \in \mathscr{B}$ and

(b) right semi-invariant if $\mu(C x) \geqq \mu(C)$, the rest as in (a).

(c) $r^{*}$-invariant if $\mu\left(B x^{-1}\right)=\mu(B)$ for all $B \in \mathscr{B}$ and $x \in S$, where $B x^{-1} \equiv\{s \in S ; s x \in B\}$

(d) right mobile if the mapping $x \rightarrow \mu(C x)$ is continuous for all compact $C$.

Analogous "left" conditions are defined similarly. The semigroup $S$ is said to satisfy condition ' $R$ ' if $A^{-1} B \equiv \cup\left\{x^{-1} B ; x \in A\right\}$ is compact whenever $A$ and $B$ are so. Condition ' $L$ ' is defined similarly replacing $A^{-1} B$ by $A B^{-1}$. We note that any topological group or a compact topological semigroup satisfies conditions ' $L$ ' and ' $R$.'

The convolution $\mu_{1} * \mu_{2}$ of two regular probability measures is defined as the unique regular probability measure induced by the linear functional on $C_{0}(S)=$ the space of continuous functions with compact 
support, defined by $I(f) \equiv \iint f(x y) \mu_{1}(d x) \mu_{2}(d y)$, the inner integral being a continuous function of $y$ by [3, p. 205]. From [3], it is also known that we can change the order of integration in the above integral. We call $\mu_{1}$ above idempotent if $\mu_{1} * \mu_{1}=\mu_{1}$.

The following converse of Haar's theorem is a consequence of a theorem of Ulam [9, Theorem 1]: If there exists a right invariant nonzero measure $\mu$ on a complete separable metric group, then the group is locally compact. One of the objects of this paper is to investigate extensions of this theorem to semitopological semigroups. Our main result in this direction is:

THEOREM 1. (a) Let $S$ be a topological semigroup satisfying condition ' $R$.' Then if $\mu$ is a nontrivial $r^{*}$-izvariant measure on $S$, then $S$ is locally compact. (b) Let $S$ be a complete separable metric semitopological semigroup and $\mu$ be a finite nonzero $r^{*}$-and $l^{*}$-invariant measure with support $S$. Then $S$ is a compact topological group. In case $S$ is topological, this result is also valid for infinite $\mu$ in the sense that $S$ will be a locally compact topological group in this case.

Part (a) of this theorem improves some of the results of [5], where $\mu$ has been assumed inner regular and $S$, a topological group. We later give examples showing that the above theorem need not be true even for finite $r^{*}$-invariant nontrivial measures on topological semigroups (complete separable metric) in the absence of condition ' $R$.'

In [10], Pym obtained a characterization of idempotent (probability) measures on compact semitopological semigroups. Our Theorem 3 extends his result to the locally compact case using DeLeeuw and Glicksberg's results on almost periodic compactifications in [4]. This also gives as a corollary (Theorem 4) a characterization of finite $r^{*}$-invariant measures on locally compact semitopological semigroups and a characterization of idempotent measures on locally compact abelian semitopological semigroups (Corollary 2). Theorem 4 for general $r^{*}$-invariant $\mu$ and topological $S$ is essentially the (still open) conjecture of [1], but is has been proved for finite $\mu$ in [7].

It is well known that a locally compact (or complete separable metric) semitopological group (i.e., an algebraic group with separately continuous multiplication $(x, y) \rightarrow x y$ ) is a topological group. (See [6] and [2, p. 36].) Our Proposition 1 presents an interesting result in this direction for semitopological groups satisfying condition ' $L$ ' from which it follows that a right (or left) invariant metric semitopological group is topological (Corollary 1).

Some of our results (e.g., the key Lemma 2 and Th. 4) have been obtained when $S$ is topological (i.e., multiplication $(x, y) \rightarrow x y$ is 
jointly continuous) in [7]. The methods of [7] do not carry over to the semitopological case since several necessary facts (e.g., the closedness of the kernel of $S$ when it exists, the continuity of $(x, y) \rightarrow(x, y x)$, etc.) used in [7] ceases to be true in the semitopological case. A counterexample showing that the kernel (= the minimal ideal) need not be closed even in a compact semitopological semigroup is given in $[2$, p. 156]. To tackle the problems on invariant measures in this paper, our main tool is Lemma 2. Finally Theorem 2 shows that admission of an (two-sided) invariant measure is equivalent to embeddability in a group even for non-abelian semigroups having the left and right translations i.e. the mappings $x \rightarrow a x$ and $x \rightarrow x a$ respectively (for every $a \in S$ ) as open mappings.

2. Before we go into the lemma leading to our main results, we present a result (Proposition 1) which might give us some insight into the conditions ' $L$ ' and ' $R$.'

Before we state and prove the proposition, let us recall from $p$. 248 of 'Topology' (Allyn \& Bacon, Inc., Boston, 1966) by Dugundji that a Housdorff space $X$ is called a $k$-space if it has the weak topology determined by the family of its compact subspaces. A set is open in this topology iff its intersection with each compact $C$ is open in $C$. Every first countable space is a $k$-space, but the cartesian product of two $k$-spaces may not be a $k$-space. (See the book by Dugundji, p. 248 and p. 249).

Proposition 1. Let $S$ be a first countable semitopological group satisfying condition ' $L$ ' or ' $R$.' Then $S$ is a topological group.

[We are thankful to the referee for the following elegant proof which replaces our original proof. It seems that the proposition may actually be valid in more general semitopological groups.]

\section{Proof. Let $S$ satisfy condition ' $L$.'}

(Continuity of the inverse). If $f$ is a function from a $k$-space $X$ into a Hausdorff space $Y$, then $f$ is contiunous iff all of its restrictions to compact subspaces are continuous. If $f$ maps compact subsets onto compact subsets and is, in addition, bijective, then its continuity follows. In view of condition ' $L$ ' this applies to any group which is a $k$-space; in particular, this applies to $S$.

(Joint continuity of multiplication). Let $C \subset S \times S$ be compact and $m: S \times S \rightarrow S$ the multiplication; we show that $m \mid C$ is continuous. Since the projections are continuous, $p r_{1} C \times p r_{2} C$ is a compact space $K$ containing $C$; it suffices to show that $m \mid K$ is continuous where 
$K=A \times A$ with compact $A$. Let $(x, y) \in A \times A$ and let $\mathscr{C}$ (resp. $\mathscr{Y}$ ) be the filter basis of all $\mathrm{cl}(A \cap U)$, where $U$ ranges through the neighborhoods of $x$ (resp. $y$ ). Then $\mathscr{W}=\{X Y:(X, Y) \in \mathscr{C} \times \mathscr{\mathscr { C }}\}$ is a filter basis, and since $X$ and $Y$ are compact, $X Y=X\left(Y^{-1}\right)^{-1}$ is compact by the continuity of the inversion and ' $L$.'

Let $d \in \cap \mathscr{W}$; then for each $i=(X, Y) \in \mathscr{L} \times \mathscr{C}$, we have elements $\left(x_{i}, y_{i}\right) \in X \times Y$ such that $d=x_{i} y_{i}$. Since $A$ is compact, hence regular, $\mathscr{C}$ (resp. $\mathscr{V}$ ) is a neighborhood basis for $x$ (resp. $y$ ) on $A$. Therefore $\lim \left(x_{i}, y_{i}\right)=(x, y)$. Then using the continuity of the inverse and left translations, we obtain $d y^{-1}=d\left(\lim y_{i}^{-1}\right)=\lim d y_{i}^{-1}=\lim x_{i}=x$ so that $d=x y$. Thus $\cap \mathscr{Y}=\{x y\}$. However, a filter basis of compact sets (in a Hausdorff space) converges iff its intersection is singleton; hence $\lim m(\mathscr{Q} \times \mathscr{C})=\lim \mathscr{W}=\varkappa y$. Thus $m \mid A \times A$ is continuous.

If $S \times S$ is a $k$-space, then, again $m$ is continuous iff $m \mid C$ is continuous for all compact $C \subset S \times S$. But since $S$ is first countable, $S \times S$ is also first countable and hence a $k$-space.

CoRollary 1. A right (or left) invariant metric semitopological group is topological.

Proof. It is sufficient to show that a semitopological group $S$ with a right invariant metric satisfies condition ' $L$ ' since the result then follows from the previous proposition. We show that for compact subsets $A$ and $B$, the set $A B^{-1}$ is sequentially compact. Let $\left\{x_{n}\right\}$ be a sequence in $A B^{-1}$ so that we can find a sequence $\left\{y_{n}\right\}$ in $B$ such that the sequence $\left\{x_{n} y_{n}\right\}$ is in $A$. Since $A$ and $B$ are sequentially compact, we can find suitable subsequences $\left\{y_{n^{\prime}}\right\}$ and $\left\{x_{n^{\prime}} y_{n^{\prime}}\right\}$ converging to $y$ in $B$ and $z$ in $A$ respectively. Now let $d$ be the invariant (right) metric on $S$. Then $d\left(x_{n^{\prime}}, z y^{-1}\right)=d\left(x_{n^{\prime}} y_{n^{\prime}}, z y^{-1} y_{n^{\prime}}\right) \leqq d\left(x_{n^{\prime}} y_{n^{\prime}}, z\right)+$ $d\left(z, z y^{-1} y_{n^{\prime}}\right)$, which tends to zero as $n^{\prime}$ tends to infinity, since by the continuity of the left translation, $z y^{-1} y_{n^{\prime}}$ converge to $z y^{-1} y=z$ and $x_{n}, y_{n^{\prime}}$ converge to $z$.

Since $r^{*}$-invariance implies right semi-invariance, the following lemma proves part (a) of Theorem 1.

LEMma 1. Let $S$ be a topological semigroup with condition ' $R$.' Let $\mu$ be a non-trivial right semi-invariant measure. Then $S$ is locally compact.

Proof. Let $x \in S$. Let $K$ be compact such that $\mu(K)=d, d>0$. Since $\mu$ is locally finite, we can find an open set $U \supset K x$ such that $\mu(U)<\infty$. Since $S$ is topological, we can find open $V$ such that $x \in V$ 
and $K V \subset U$. We claim that $\mathrm{cl}(V)$ is compact. Suppose not. If for every $y \in V, K y \cap K x$ is nonempty, then $V \subset K^{-1}(K x)$ which is compact by ' $R$ ' and this contradicts our claim. Therefore, there is $y_{1} \in V$ such that $K y_{1} \cap K x$ is empty. Similarly, we can find $y_{2}$ in $V$ such that $K y_{2} \cap\left(K x \cup K y_{1}\right)$ is empty. This process must terminate after a finite number of times since $\mu(U)<\infty$. Hence $\mathrm{cl}(V)=$ compact.

If $S$ was a topological group, we only needed $\mu$ to be right mobile. The following lemma is our key lemma.

Lemma 2. Let $S$ be a complete metric (or locally compact) semitopological semigroup and $\mu$ be a finite regular $r^{*}$-and $l^{*}$-invariant measure with support $S$. Then $S$ is a compact group.

Proof. First we observe that since $\mu$ is $r^{*}$ - and $l^{*}$-invariant with support $S, \operatorname{cl}(S x)=S=\operatorname{cl}(x S)$ for every $x$ in $S$, (see [1]). So if $S$ was compact, $S$ will be algebraically a group and therefore a topological group by Ellis' theorem [2, p. 36] and the lemma would be trivial. The non-triviality of the lemma lies in the fact that it is not at all clear that the translations in $S$ are closed in our lemma.

It is clear from above that when the translations on $S$ are closed, $S$ is a group. In the metric case, $S$ being the support of a regular measure contains a sigma-compact set whose complement is a set of measure zero and therefore $S$ is separable. So then $S$ will be a complete separable metric (or locally compact) semitopological group and hence a topological group by [6]. The lemma will then follow from Lemma 1.

Since $\mathrm{cl}(S e)=S e$ and $\mathrm{cl}(e S)=e S$ when $e$ is an idempotent and since $\operatorname{cl}(S x)=\operatorname{cl}(x S)=S$ for every $x$ in $S$, it suffices to show that for every $x$ in $S$, both $S x$ and $x S$ contain an idempotent. We do this in the following steps.

(1) For $a \in S, a S a$ is a bicancellative semitopological semigroup, since $\operatorname{cl}(S a)=S=\operatorname{cl}(a S)$. (See [7].)

(2) Let $K$ be a compact set $\subset a S a$. Let $\theta(x, y)=(x, y x)$. Since $S$ is semitopological, it is not clear that $\theta(K \times K)$ is closed in $a S a \times a S a$. We show that $\theta(K \times K)$ is closed in $a S a \times a S a$ and this is done in two sub-steps.

Sub-step I. The weakly almost periodic continuous functions on [aSa separate points of aSa. [A function $f$ in $C_{b}(S)=$ the bounded continuous functions on $S$, is weakly almost periodic if $\left\{R_{y} f ; y \in S\right\}$ is conditionally weakly compact in $C_{b}(S)$, where $\left[R_{y} f\right](s) \equiv f(s y)$.]

Let $U=\left\{f:|f| \leqq n, f \in L_{2}(S, \mu)\right\}$ where $n$ is some fixed natural number and $L_{2}(S, \mu)$ is the Hilbert space of equivalence classes of 
square-integrable functions. Then $U$ is weakly compact in $L_{2}$. For each $f$ in $U$, let $[T f](x) \equiv \int f(s x) g(s) \mu(d s)$, where $g$ is some fixed function in $C_{b}(S)$. When $S$ is locally compact, the continuity of [Tf] follows from [3, 1.2]. When $S$ is metric, $S$ is first countable so that the standard dominated convergence theorem implies the continuity of $T f$. Since $\mu$ is invariant, it is easy to see that $T$ is a continuous linear mapping from $U$ (with relative strong topology from $L_{2}$ ) into $C_{b}(a S a)$ (restriction of $T f$ to $a S a$ ), with strong topology. In fact, the domain of $T$ can be taken as $L_{2}$, and hence $T$ is also continuous from $L_{2}$ (with weak topology) into $C_{b}(a S a)$ (with weak topology). Now we observe that

$$
\begin{aligned}
{\left[R_{y} T f\right](x)=[T f](x y) } & =\int f(s x y) g(s) \mu(d s)=\int\left[R_{y} f\right](s x) g(s) \mu(d s) \\
& =\left[T R_{y} f\right](x)
\end{aligned}
$$

which means that $T f$ is weakly almost periodic because of the weak continuity of $T$.

Now let $s, s^{\prime} \in a S a, s \neq s^{\prime}$. Then for $y \in a S a, s y \neq s^{\prime} y$ by (1). Hence there is a nonnegative $f \in C_{b}(S)$ such that $f(s y)>f\left(s^{\prime} y\right)+1$. Now the set $V^{\prime}=\left\{x \in S: f(s x)>f\left(s^{\prime} x\right)+1\right\}$ is a nonempty open set in $S$. Let $A$ be compact, $A \subset V^{\prime}$ and $\mu(A)>0$ and let $g$ be a nonnegative function in $C_{b}(S)$ such that $g(x)=1$ for $x \in A, f(x)=0$ for $x \notin V^{\prime}$. Then $[T f](s)-[T f]\left(s^{\prime}\right)>0$. This proves Sub-step I.

Sub-step II. $\theta(K \times K)$ is closed in $a S a \times a S a$.

We consider the weakly almost periodic compactification $S^{w}$ of $a S a$. (See [4, p. 82].) $S^{w}$ is a compact semitopological semigroup and there is a continuous homomorphism $p: a S a \rightarrow S^{w}$ such that $p(a S a)$ is dense in $S^{w}$. Because of Sub-step I, $p$ is also $1-1$. Now we restrict $\mu$ to the Borel sets of $a S a$ (in the relative topology). We also notice that if $K$ is compact in $S$, then $a S a \supset a K a$ and $\mu(a K a)=$ $\mu\left(a^{-1} a K a a^{-1}\right) \geqq \mu(K)$ since $\mu$ is invariant and $K \subset a^{-1} a K a a^{-1}$. This means that we can find a $\sigma$-compact set $B \subset S$ such that $\mu(a B a)=\mu(S)$, since $\mu$ is regular. So we can now define a nonzero finite measure $\mu^{w}$ on $S^{w}$ by $\mu^{w}(H) \equiv \mu\left(p^{-1}(H)\right)$ for every Borel $H \subset S^{w}$. Then $\mu^{w}$ is inner regular relative to compact sets (and hence outer regular relative to open sets since $\mu^{w}$ is finite); for, $\mu$ is regular and if $K \subset p^{-1}(H)$, then $p(K) \subset H$ and $\mu^{w}(H-p(K))=\mu\left(p^{-1}(H)-K\right)$, since $p$ is $1-1$. It follows easily that $\mu^{w}$ has support $S^{w}$ and for every $z$ in $p(a S a)$ and every Borel set $B$ in $S^{w}, \mu^{w}\left(B z^{-1}\right)=\mu^{w}(B)=\mu^{w}\left(z^{-1} B\right)$.

Since by [3, 1.2], the functions $y \rightarrow \int f(x y) \mu^{w}(d x)$ and $y \rightarrow \int f(y x) \mu^{w}(d x)$ are continuous on $S^{w}$, it follows as usual (as in [7]) that the functions 
$y \rightarrow \mu^{w}\left(C y^{-1}\right)$ and $y \rightarrow \mu^{w}\left(y^{-1} C\right)$ are upper semi-continuous for compact $C$. Hence using upper semi-continuity of these functions, we see that for every compact $C$ in $S^{w}$ and every $x$ in $S^{w}, \mu^{w}\left(C x^{-1}\right) \geqq \mu^{w}(C)$ and $\mu^{w}\left(x^{-1} C\right) \geqq \mu^{w}(C)$. Now if $y \in S^{w}$ and $y \notin S^{w}$ for some $x$, then there is a compact neighborhood $V$ of $y$ such that $V x^{-1}$ is empty which contradicts that $\mu^{w}\left(V x^{-1}\right) \geqq \mu^{w}(V)>0$. Hence for every $x$ in $S^{w}, S^{w} x=S^{w}$. Similarly, $x S^{w}=S^{w}$. Hence $S^{w}$ is a group so that $S^{w}$ is a topological group, by Ellis' theorem. (See [2, p.36]。)

Let $\left\{s_{j}\right\}$ and $\left\{t_{j}\right\}$ be two nets in the compact set $K$ such that $s_{j}$ converges to $s_{0}$ and $t_{j}$ converges to $t_{0}$. Also let the net $\left\{s_{j} t_{j}\right\}$ converges to some point $v$ in $a S a$. Since $S^{w}$ is topological, $p\left(s_{j} t_{j}\right)=p\left(s_{j}\right) p\left(t_{j}\right)$ converges to $p\left(s_{0}\right) p\left(t_{0}\right)=p\left(s_{0} t_{0}\right)$. So $s_{0} t_{0}=v$, since $p$ is $1-1$. It is now easy to see that $\theta(K \times K)$ is closed.

(3) By the argument preceding (1), the lemma will be proved if we show that for every $a$ in $S, a S a$ contains an idempotent. This can be shown now by following Lemma 2.3 in [7]. For the sake of completness, we briefly outline the arguments.

Let $\mu(S)=1$. Let $a \in S$. Then we can find compact $K$ in $S$ such that $\mu(a K a)>.99$, by regularity and invariance of $\mu$. We extend the usual product measure $\mu \times \mu$ on $a K a \times a K a$ to a unique regular measure $m$ on the Borel subsets of $a K a \times a K a$. Let us define $\theta(x, y)=(x, y x)$ and $\pi(x, y)=(y, x)$. Let $D=(a K a \times a K a) \cap \theta(a K a \times$ $a K a)$. Then by Sub-step II above, $D$ is compact in the product space. Using Fubini's theorem, it follows that $m(D)>$.8. Since $\pi$ is measurepreserving, $\pi(D) \cap D$ is nonempty. Hence there are $x, y, u, v$ in $a K a$ such that $(x, y x)=(v u, u)$ so that $x=v u, y x=u$ and $(v y)(v y) x=(v y) x$. By cancellation in $a S a, v y$ is an idempotent in $a S a$. This proves Lemma 2.

Proof of Theorem 1. The proof of part (a) and first part of part (b) follows from Lemma 1 and Lemma 2. The last part of part (b) follows from [11] and Lemma 1.

REMARK 0. The proof of Lemma 2 can also proceed along the following lines. Consider the mapping $\pi: a S a \rightarrow \mathscr{L}\left(L_{2}(S, \mu)\right)$ defined by $[\pi(t) f](s) \equiv f^{\prime}(s t)$, where $\mathscr{L}$ is the space of bounded linear operators on $L_{2}(S, \mu)$, the Hilbert space of equivalence classes of square-integrable functions. Then since for every $t \in a S a$, we have $\|\pi(t)\|=1$ (where $\|\cdot\|$ is the usual operator-norm), $\overline{\pi(a S a)}$, the closure of $\pi(a S a)$ in the weak operator topology of $\mathscr{L}$, is compact. By a similar kind of argument as in $[4, T h .3 .1$, p. 72], it follows that $\overline{\pi(a S a)}$ is a compact semitopological semigroup. Also as in the proof of Sub-step I, the mapping $\pi^{\prime}: a S a \rightarrow \overline{\pi(a S a)}$, defined by $\pi$, becomes continuous. Also $\pi^{\prime}$ is injective since by $[2$, p. 96$], \pi\left(t_{1}\right)=\pi\left(t_{2}\right)$ iff $x t_{1}=x t_{2}$ for every $x \in S$ 
and $a S a$ is bicancellative [11, p. 262]. Now the proof of Sub-step II can be continued replacing $S^{w}$ by $\overline{\pi(a S a)}$ and the mapping $p$ by the mapping $\pi^{\prime}$.

REMARK 1. In Lemma 1, if $\mu$ was finite, we could assume $S$ to be only semitopological and conclude that $S$ was compact. But in Lemma 1 , if $S$ is a topological group, a finite mobile measure will make $S$ only locally compact, not compact. For, if $S=(0, \infty)$, the positive reals (multiplicative) and $\mu$, the Stieltjes measure induced by $x \rightarrow[x / x+1]$, then $\mu$ is a finite mobile measure whereas $(0, \infty)$ is not compact.

REMARK 2. We know that a Haar measure on a topological group makes it locally compact (by Lemma 1 ) and hence $\mu$ is regular when restricted to $\sigma$-bounded sets. (See Halmos' Measure theory, p. 288.) This is no longer true in the nongroup situation. A nonzero $r^{*}$-invariant measure need not be even nontrivial even if its support is a topological left group. For example, let $S=E \times G$, where $G$ is a compact metric group and $E$, a subset of $[0,1]$ such that $P^{*}(E)=1$ and $P_{*}(E)=0$, where $P^{*}$ and $P_{*}$ are the outer and inner Lebesgue measure respectively. We define multiplication on $S$ by $(e, g)\left(e^{\prime}, g^{\prime}\right)=\left(e, g g^{\prime}\right)$. Then $S$ is a separable metric topological (with product topology) left group. Let $\mu=P^{*} \times \pi, P^{*}$ being a measure on the Borel sets of $E$ and $\pi$, the normed Haar measure of $G$. Let us consider $\{B: B$ is a Borel subset of $E \times G$ and $\mu\left(B x^{-1}\right)=\mu(B)$ for every $x$ in $\left.E \times G\right\}$ which is a sigma-class (see [8] for definition) containing all the measurable rectangles and hence by the theorem on p. 72 in [8], it contains the smallest sigma-algebra containing the measurable rectangles which means $\mu$ is an $r^{*}$-invariant Borel measure on $E \times G$. Since $P^{*}$ is zero on the compact sets of $E, \mu$ is zero on all compact sets of $E \times G$, by Fubini's theorem. But clearly, the support of $\mu$ is all of $E \times G$.

REMARK 3. The converse of Haar's theorem need not be true for $r^{*}$-invariant measures. For example, in Remark 2 , replace $E$ by $\times_{i=1}^{\infty} E_{i}$, where $E_{i}=[0, \infty)$ for every $i$ and take $\mu=P \times \pi$, where $P=\prod_{i=1}^{\infty} P_{i}$, each $P_{i}$ being the regular probability measure induced by the function $x \rightarrow[x / x+1]$ on $[0, \infty)$. Then as in Remark $2, \mu$ becomes an $r^{*}$-invariant regular probability measure with support $E \times G$, a complete separable metric nonlocally compact topological left group.

3. In this section we will present a few results which might help us understand relations between different invariant measures and some suitable Haar measure. We observe that the three types of invariance defined in Section 1 are all equivalent in a left group 
since every element in a left group has a left inverse with respect to a fixed idempotent which is a right identity. This is true in a more general situation as the following proposition shows. (Compare Theorem 4.8, p. 93, in [2].)

Proposition 2. A locally compact semitopological semigroup $S$ having a minimal left and a minimal right ideal admits a right semi-invariant measure with non-empty support $F$ iff the kernel $K$ of $S$ is a closed left group and the measure restricted to $F$ is of the form $P \times \pi, P$ being a regular measure and $\pi$ being a right Haar measure.

Proof. Suppose $S$ has a minimal left and a minimal right ideal. Then $S$ has a kernel $K$ which is completely simple. If $e$ is an idempotent in $K$, then $K e(=S e)$ is a left group. Also $F \subset S e$, as in [2, p. 92, Th. 4.6]. Since $F$ is a right ideal of $S e$, a left group, $F$ must be a left group. Then for any $s \in S, f \in F, F f s=F s=F$, since $f s \in F$ and $F$ is left simple. Hence $F \subset \cap\{S x: x \in S\}=L$, which is a minimal left ideal and also a right since $L \supset F$ and $F$ is a right ideal. Hence $L=K=S e$. The rest of the theorem follows from [2, p. 94, Th. 4.9]. (Note that 'right sub-invariant' and 'right semi-invariant' are equivalent with respect to compact sets.)

The next theorem shows under what conditions an invariant measure on a locally compact semitopological semigroup $S$ is the restriction on $S$ of a Haar measure on a locally compact group containing $S$.

THEOREM 2. A locally compact right reversible (i.e. $S x \cap S y \neq \varnothing$, for every $x, y$ in $S$ ) semitopological semigroup $S$ having the left and right translations open ${ }^{1}$ can support a right and left invariant measure iff $S$ can be topologically embedded as an open subspace in a locally compact topological group and the invariant measure on $S$ is the restriction of a unimodular Haar measure on the group.

Proof. The proof, though slightly tricky, will be omitted for brevity. We will only mention that $S$ easily turns out to be bicancellative because of the invariant measure and open translations and then easily the desired embedding can be obtained by Theorem 3 in the joint note (of the authors) in Semigroup Forum, Vol. 2 (1971) p. 71, 'A note on the embedding of topological semigroups.'

We remark that $R$. Rigelhof has also obtained a similar theorem on locally compact abelian topological semigroups (Proc. Amer. Math.

1 i.e., the mappings $x \rightarrow x a$ and $x \rightarrow a x, a \in S$, are open. 
Soc., 28 (1971), 173-75).

4. The following Lemma will be used in the sequel (proof of Theorem 3). This lemma is subsumed under a more general result proven in [1, p. 378] (see also [2, p. 94, Theorem 4.9]).

LEMma 3. Let $\mu$ be an $r^{*}$-invariant regular probility measure with support $S$, a locally compact topological left group (i.e., $S x=S$ for all $x \in S$ and $S$ is right cancellative). Then $E=$ the set of idempotents of $S$, is nonempty and $G \equiv e S$ is a topological group for $e \in E$. Also there is a probability measure $\mu_{1}$ with support in $E$ and a normed Haar measure $\mu_{2}$ with support $G$ such that $\mu=\mu_{1} * u_{2}$.

Now let us consider an idempotent probability measure $\mu$ on a locally compact semitopological semigroup $S$. These measures have been studied in the compact case by many mathematicians including Glicksberg [3, Theorem 4.2] and Pym [10, the main theorem]. They have been completely characterized on locally compact (or complete metric) topological semigroups by the authors in [7]. Let $F$ be the support of $\mu$. Then by following the proof of Theorem 3.1 in [7], one easily obtains, using Lemma 2, a completely simple kernel $K \subset F, K \supset$ $F F$ and $\mathrm{cl}(F F)=\overline{F F}=F$. Also it follows from [7] that for every Borel set $B$ and $x, y$ in $F, \mu\left(B x^{-1} y^{-1}\right)=\mu\left(B x^{-1}\right)$ and $\mu\left(x^{-1} B\right)=\mu\left(y^{-1} x^{-1} B\right)$. This means that for any other regular probability measure $P$, we have $\mu * P * \mu=\mu$. Let $e$ be an idempotent in $K$. Then $K e=F e$, a topological left group; also $e K=e F$ is a topological right group; and $e K e=e F e$, a topological group. (These follow since $K e, e K$ and $e K e$ are all closed and so locally compact so that Ellis' theorem applies.) Let $E=$ the idempotents of $K e$ and $E^{\prime}=$ the idempotents in $e K$ and $G=e K e$. We define $\mu_{e}(B)=\mu\left(B e^{-1}\right)$ and $e_{e} \mu(B)=\mu\left(e^{-1} B\right)$. Then $\mu_{e}$ and $e_{e} \mu$ are regular probability measures with supports $K e$ and $e K$ respectively. Also $\mu=\mu_{e} *_{e} \mu$ and $\mu_{e}$ is $r^{*}$-invariant on its support $F e$ and $e_{e} \mu$ is $l^{*}$-invariant, on its support $e F$. By Theorem 4.9, p. 94 in [2], there are regular probability measures $\mu_{1}$ and $\mu_{2}$ (a normed Haar measure) with supports respectively $E$ and $G$ such that $\mu_{e}=$ $\mu_{1} \times \mu_{2}$ on $E \times G$, which is topologically isomorphic to $K e$. We regard $\mu_{1}$ and $\mu_{2}$ as measures on $F$. Then by Lemma 3, we have $\mu_{e}=\mu_{1} * \mu_{2}$. Similarly we can find a regular probability measure $\mu_{3}$ with support $E^{\prime}$ such that $\mu_{2} * \mu_{3}={ }_{e} \mu$. Hence it follows that $\mu=\mu_{1} * \mu_{2} * \mu_{3}$, since $\mu_{2}$ is idempotent, being a Haar measure. Since $\mu_{3} * \mu_{1}$ has support contained in the support of $\mu_{2}$ and $\mu_{2}$ is a Haar measure, $\mu_{2} *\left(\mu_{3} * \mu_{1}\right)=$ $\mu_{2}$ so that every measure of the form $\mu_{1} * \mu_{2} * \mu_{3}$ is idempotent. Hence

2 By a completely simple kernel $K$, we mean a minimal ideal containing a primitive idempotent. Necessary facts on such kernels which are used in this paper are given in $[2]$. 
we have proved

THEOREM 3. Let $\mu$ be a regular idempotent probability measure on a locally compact semitopological semigroup. If $F$ is the support of $\mu, F$ contains a dense completely simple kernel $K$ and $\mu$ can be expressed in the form $\mu_{1} * \mu_{2} * \mu_{3}$ where $\mu_{1}$ has support $E$ (the idempotents in $K e), \mu_{2}$ is the normed Haar measure on eKe and $\mu_{3}$ has support $E^{\prime}$ (the idempotents in eK), where $e$ is a fixed idempotent in $K$. Conversely, every measure of the form $\mu_{1} * \mu_{2} * \mu_{3}$ where $\mu_{2}$ is a Haar measure whose support contains the support of $\mu_{3} * \mu_{1}$, is idempotent.

COROLlaRY 2. Let $\mu$ be a regular idempotent probability measure on a locally compact abelian semitopological semigroup. Then $\mu$ is a normed Haar measure on its support $F$, which is a compact group.

Proof of the corollary. From Theorem 3, the completely simple kernel $K$, being abelian, is a group. Hence if $e$ is an idempotent in $K, K=K e=F e$. But $F e$ is closed in $F$. So $K=F$, since $K$ is dense in $F$. Since $e K e=K=F, \mu$ becomes $\mu_{2}$ in Theorem 3 .

We remark that this results extends the main result of Tserpes and Sun in [12].

Finally, we give a characterization of $r^{*}$-invariant measures on a locally compact semitopological semigroup $S$.

THEOREM 4. Let $\mu$ be a finite $r^{*}$-invariant measure (regular) on a locally compact semitopological semigroup $S$. Then $F$, the support of $\mu$ is a left group (see Lemma 3), and $\mu$ is of the form $\mu_{1} * \mu_{2}$ where $\mu_{1}$ is a measure with its support contained in $E=$ the idempotents of $F$ and $\mu_{2}$ is the Haar measure on $e F$, a compact group for some idempotent $e$ in $E$. Conversely, every measure of the form $\mu_{1} * \mu_{2}$ is invariant.

Proof. Since $\mu$ is $r^{*}$-invariant, cl $(F a)=F$ for every $a$ in $F$. (See [1].) We normalise $\mu$, since this does not change its support. Then for every Borel set $B, \mu(B)=\int \mu\left(B x^{-1}\right) \mu(d x)$ so that $\mu$ is idempotent. By Theorem $3, F$ has a dense completely simple kernel $K$. Let $e \in E$. Then $F=\operatorname{cl}(F e)=F e=K e$, if $e$ is in $K$, since $K$ is an ideal of $F$ and so $F e \subset K, F e=F e e \subset K e$. But $K e$ is a left group, since $K$ is completely simple. (See [2].) The rest of the theorem follows from Lemma 3.

We now close the paper with the following remark.

REMARK 4. We conjecture that Lemma 2 (compactness in the 
conclusion replaced by local compactness) and Theorem 4 are both true for infinite measures. A partial solution is given by the following theorem whose proof appears in a paper of the authors "A problem on $r^{*}$-invariant measures on locally compact semigroups" Indiana University Math. J., 21 (1972), 973-977.

THEOREM 5. Let $\mu$ be an $r^{*}$-invariant measure (not necessarily finite) on a locally compact topological semigroup $S$. Then $F$, the support of $\mu$ is a left group if and only if $\mu$ is right invariant on its support i.e., $\mu(K x)=\mu(K)$ for every compact $K \subset F$ and $x \in F$.

The authors wish to express their indebtedness to the referee for some helpful remarks and in particular the alternate elegant proof of Proposition 1.

\section{REFERENCES}

1. L. N. Argabright, A note on invariant integrals on locally compact semigroups, Proc. Amer. Math. Soc., 17 (1966), 377-382.

2. J. F. Berglund and K. H. Hofmann, Compact semitopological semigroups and weakly almost periodic functions, Lecture Notes in Math., no. 42, Springer-Verlag, Berlin and New York, 1967.

3. I. Glicksberg, Weak compactness and separate continuity, Pacific J. of Math., 11 (1961), 205-214.

4. K. DeLeeuw and I. Glicksberg, Applications of almost periodic compactifications, Acta. Math., 105 (1961), 63-98.

5. C. Gowrisankaran, Radon measures on groups, Proc. Amer. Math. Soc., 25 (1970), 381-384.

6. D. Montgomery, Continuity in topological groups, Bull. Amer. Math. Soc., 42 (1936), 879-882.

7. A. Mukherjea and N. A. Tserpes, Idempotent measures on locally compact semigroups, Proc. Amer. Math. Soc., 29 (1971), 143-150.

8. T. Neubrunn, A note on quantum probability spaces, Proc. Amer. Math. Soc., 25 (1970), 672-675.

9. J. C. Oxtoby, Invariant measures in groups which are not locally compact, Trans. Amer. Math. Soc., 60 (1946), 215-237.

10. J. S. Pym, Idempotent probability measures on compact semitopological semigroups, Proc. Amer. Math. Soc., 21 (1969), 499-501.

11. N. A. Tserpes and A. Mukherjea, On certain conjectures on invariant measures on semigroups, Semigroup Forum, 1 (1970), 260-266.

12. N. A. Tserpes and T. C. Sun, Idempotent probability measures on locally compact abelian semigroups, J. Math. Mech., 19 (1970), 1113-1116.

Received August 24, 1971 and in revised form Junuary 28, 1972.

UNIVERSITY OF SOUTH FLORIDA 


\section{PACIFIC JOURNAL OF MATHEMATICS}

\section{EDITORS}

H. SAMELSON

Stanford University

Stanford, California 94305

C. R. Hовву

University of Washington

Seattle, Washington 98105
J. DugundJI

Department of Mathematics University of Southern California

Los Angeles, California 90007

RICHARD ARENS

University of California

Los Angeles, California 90024

\section{ASSOCIATE EDITORS}

E. F. BECKENBACH

B. H. NeumanN

F. WOLF

K. YoSHIDA

\section{SUPPORTING INSTITUTIONS}

UNIVERSITY OF BRITISH COLUMBIA

CALIFORNIA INSTITUTE OF TECHNOLOGY

UNIVERSITY OF CALIFORNIA

MONTANA STATE UNIVERSITY

UNIVERSITY OF NEVADA

NEW MEXICO STATE UNIVERSITY

OREGON STATE UNIVERSITY

UNIVERSITY OF OREGON

OSAKA UNIVERSITY
UNIVERSITY OF SOUTHERN CALIFORNIA

STANFORD UNIVERSITY

UNIVERSITY OF TOKYO

UNIVERSITY OF UTAH

WASHINGTON STATE UNIVERSITY

UNIVERSITY OF WASHINGTON

AMERICAN MATHEMATICAL SOCIETY

NAVAL WEAPONS CENTER

Printed in Japan by International Academic Printing Co., Ltd., Tokyo, Japan 


\section{Pacific Journal of Mathematics}

\section{Vol. 44, No. $1 \quad$ May, 1973}

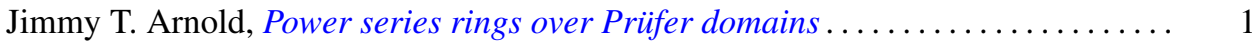

Maynard G. Arsove, On the behavior of Pincherle basis functions . . . . . . . . . 13

Jan William Auer, Fiber integration in smooth bundles ................. 33

George Bachman, Edward Beckenstein and Lawrence Narici, Function algebras

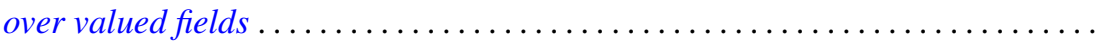

Gerald A. Beer, The index of convexity and the visibility function . . . . . . . . . . .

James Robert Boone, A note on mesocompact and sequentially mesocompact

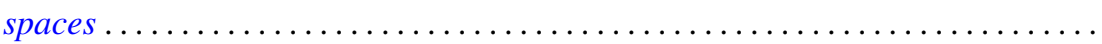

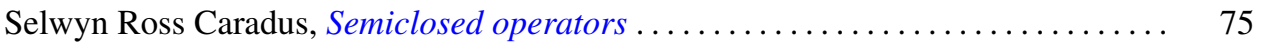

John H. E. Cohn, Two primary factor inequalities . . . . . . . . . . . . . . . 81

Mani Gagrat and Somashekhar Amrith Naimpally, Proximity approach to

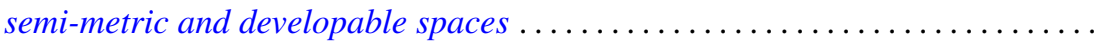

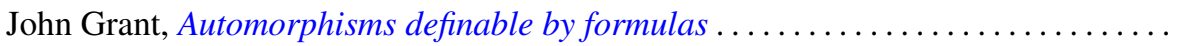

Walter Kurt Hayman, Differential inequalities and local valency ..............

Wolfgang H. Heil, Testing 3-manifolds for projective planes . . . . . . . . . . . . .

107

Melvin Hochster and Louis Jackson Ratliff, Jr., Five theorems on Macaulay

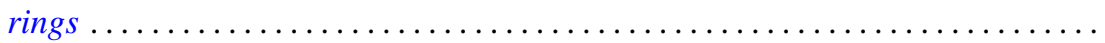

Thomas Benton Hoover, Operator algebras with reducing invariant subspaces ....

James Edgar Keesling, Topological groups whose underlying spaces are separable

Fréchet manifolds...

Frank Leroy Knowles, Idempotents in the boundary of a Lie group . .

191

George Edward Lang, The evaluation map and EHP sequences ...

201

Everette Lee May, Jr, Localizing the spectrum . . . . . . . . . . . .

211

Frank Belsley Miles, Existence of special $K$-sets in certain locally compact abelian groups.

Susan Montgomery, A generalization of a theorem of Jacobson. II . .

T. S. Motzkin and J. L. Walsh, Equilibrium of inverse-distance forces in

three-dimensions.

Arunava Mukherjea and Nicolas A. Tserpes, Invariant measures and the converse

of Haar's theorem on semitopological semigroups .

James Waring Noonan, On close-to-convex functions of order $\beta$

Donald Steven Passman, The Jacobian of a growth transformation

Dean Blackburn Priest, A mean Stieltjes type integral ........ .

Joe Bill Rhodes, Decomposition of semilattices with applications to topological

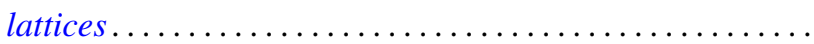

Claus M. Ringel, Socle conditions for $\mathrm{QF}-1$ rings ..........

Richard Rochberg, Linear maps of the disk algebra

Roy W. Ryden, Groups of arithmetic functions under Dirichlet convolution . .

Michael J. Sharpe, A class of operators on excessive functions

Erling Stormer, Automorphisms and equivalence in von Neumann algebras ..

Philip C. Tonne, Matrix representations for linear transformations on series

analytic in the unit disc. 